\title{
Carney triad can be (rarely) associated with germline succinate dehydrogenase defects
}

\author{
Sosipatros A Boikos ${ }^{1,7,8}$, Paraskevi Xekouki ${ }^{1,7}$, Elena Fumagalli ${ }^{2}$, Fabio R Faucz ${ }^{\star, 1}$, Margarita Raygada ${ }^{1}$, \\ Eva Szarek ${ }^{1}$, Evan Ball ${ }^{1}$, Su Young Kim ${ }^{3}$, Markku Miettinen ${ }^{4}$, Lee J Helman ${ }^{3}$, J Aidan Carney ${ }^{5}$, Karel Pacak ${ }^{6}$ \\ and Constantine A Stratakis ${ }^{\star}$
}

Carney triad, the association of paragangliomas/pheochromocytomas, gastrointestinal stromal tumors and pulmonary chondromas, is a sporadic condition that is significantly more frequent in females; its genetic etiology remains unknown. Carney triad is distinct from the dyad of paragangliomas/pheochromocytomas and gastrointestinal stromal tumors, known as Carney-Stratakis syndrome, which is inherited in an autosomal- dominant manner and is almost always caused by succinate dehydrogenase subunit mutations. In the present study, we investigated the largest cohort of Carney triad patients that is available internationally: 63 unrelated patients. Six patients (9.5\%) were found to have germline variants in the SDHA, SDHB or SDHC genes. All six patients, except one, had multifocal gastrointestinal stromal tumors, chondromas and/or paragangliomas. A patient with Carney triad and $S D H C$ variant had a ganglioneuroma. One of the patients with Carney triad and $S D H B$ mutation had a nephew with the same sequence defect, who developed a neuroblastoma. Other relatives, carriers of the identified $S D H A, S D H B$ or SDHC mutations, have not developed any of the components of Carney triad or Carney-Stratakis syndrome. None of the other 57 Carney triad patients had any genomic defects of SDHA, SDHB or SDHC genes. We conclude that, in rare occasions, Carney triad can be allelic to Carney-Stratakis syndrome. Although for the vast majority of patients with Carney triad the causative defect(s) remain(s) unknown, testing for $S D H A, S D H B$ or $S D H C$ variations should be offered, as carriers may develop isolated paragangliomas/pheochromocytomas and occasionally other tumors.

European Journal of Human Genetics (2016) 24, 569-573; doi:10.1038/ejhg.2015.142; published online 15 July 2015

\section{INTRODUCTION}

Carney triad (CTr) describes the association of paragangliomas (PGL)/ pheochromocytomas (PHEO) with gastrointestinal stromal tumors (GISTs) and pulmonary chondromas (CHOs). ${ }^{1,2} \mathrm{CTr}$ is a nonhereditary, sporadic condition, primarily seen in young women. ${ }^{3}$ Most CTr patients present with GIST and pulmonary CHO; PGL/ PHEO is rarely the presenting tumor but most often found at screening, in addition to other lesions, such as adrenocortical tumors. ${ }^{4}$ A marked decrease in the enzymatic activity of succinate dehydrogenase $(\mathrm{SDH})$ has been detected in tumors associated with $\mathrm{CTr}$, but in the first cohort of $37 \mathrm{CTr}$ patients studied by Matyakhina et $a l^{5}$ no mutations of the genes coding for the four SDH subunits (SDHA, SDHB, SDHC and SDHD, respectively, collectively known as the ' $S D H x$ ' genes) were found.

Instead, the investigation led to the description of a related disease, the dyad of PGL/PHEO and GIST, also known as Carney-Stratakis syndrome (CSS), which affects males and females equally, is almost always inherited as an autosomal-dominant trait, and is never associated with $\mathrm{CHO}^{6}$ Soon thereafter, $\mathrm{SDH} x$ sequencing defects were found to be the cause of CSS. ${ }^{6,7}$ A large number of patients presenting with isolated GIST were also found to have germline $S D H x$ defects but not necessarily CSS, ${ }^{8}$ whereas $S D H B, S D H C$ and SDHD mutations may also cause the hereditary-isolated PGL/PHEO syndromes PGL4, PGL3 and PGL1, respectively. ${ }^{9}$ A similar condition, PGL2, is caused by mutations in a gene that is associated with the maintenance of proper SDH activity, SDHAF2 (SDH5). ${ }^{10}$ SDHA defects have been found in both CSS and isolated GIST, but rarely in isolated PGL/PHEO. ${ }^{11}$

To date, the genetic $\operatorname{defect}(\mathrm{s})$ causing $\mathrm{CTr}$ remain(s) unknown. ${ }^{12}$ CTr-associated PGL/PHEO, GIST and CHO fail to express the SDHB protein, similar to tumors caused by $S D H B$ and other $S D H x$ variations $s^{8,12}$ providing a rapid diagnostic tool for their identification. ${ }^{13}$ Recently, SDHC locus-specific methylation was found to be present in CTr tumors, ${ }^{14}$ a defect that was also later described in GISTs that lack SDHB immunoreactivity but have no variations in the SDHx genes. ${ }^{15}$ This was a distinct defect, separate from the overall increased methylation of the genome seen in lesions that bear SDHx defects. ${ }^{16,17}$ Thus, SDHB deficiency by immunohistochemistry (IHC)

\footnotetext{
${ }^{1}$ Section on Endocrinology and Genetics (SEGEN), Program on Developmental Endocrinology and Genetics (PDEGEN), Eunice Kennedy Shriver National Institute of Child Health and Human Development (NICHD), National Institutes of Health (NIH), Bethesda, MD, USA; ${ }^{2}$ Adult Mesenchymal Tumour and Rare Cancer Medical Oncology Unit, Cancer Medicine Department, Istituto Nazionale Tumori, Milano, Italy; ${ }^{3}$ Pediatric Oncology Branch (POB), National Cancer Institute (NCI), NIH, Bethesda, MD, USA; ${ }^{4}$ Department of Diagnostic Pathology, National Cancer Institute (NCI), NIH, Bethesda, MD, USA; ${ }^{5}$ Laboratory of Pathology, Mayo Clinic, Rochester, MN, USA; ${ }^{6}$ Section on Neuroedocrinology (SNE), Program on Reproductive and Adult Endocrinology, NICHD, NIH, Bethesda, MD, USA

${ }^{*}$ Correspondence: Dr FR Faucz or Dr CA Stratakis, Section on Endocrinology and Genetics (SEGEN), Program on Developmental Endocrinology and Genetics (PDEGEN), Eunice Kennedy Shriver National Institute of Child Health and Human Development (NICHD), National Institutes of Health (NIH), 10 Center Drive, Building 10, NIH-Clinical Research Center, Room 1-3330, MSC1103, Bethesda, MD 20892, USA. Tel: +1 301496 4686; Fax: +1 301 4020574; E-mail: fabio.faucz@nih.gov or stratakc@mail.nih.gov ${ }^{7}$ These authors contributed equally to this work.

${ }^{8}$ Current address: Sarcoma and Rare Tumors Program, Division of Hematology, Oncology and Palliative Care, Virginia Commonwealth University, Massey Cancer Center, Richmond, VA, USA.

Received 10 March 2015; revised 15 May 2015; accepted 27 May 2015; published online 15 July 2015
} 
and SDHC gene-specific methylation provide the most recent means of identification of a CTr-associated tumor, in the absence of a known causative genetic defect.

In the present study, we investigated the largest available, international cohort of 63 patients with CTr for germline variations and/or deletions in the SDHx genes; we included here the previously studied 37 patients and resequenced them all, and, in addition, other genomic investigations were performed. Six patients were found to have germline sequence variants in $S D H A, S D H B$ or $S D H C$; one patient who was previously classified as CSS was found to have $\mathrm{CTr}$, as a $\mathrm{CHO}$ was confirmed by biopsy. All six patients, except one, had multifocal gastric GIST and pulmonary CHOs at diagnosis, whereas one had only $\mathrm{CHO}$; two developed PGL, confirmed by the biopsy. One of the patients with $C T r$ and an $S D H B$ variant had a nephew with the same sequence defect and a neuroblastoma (NB), confirming a previously reported association of $\mathrm{NB}$ with $\mathrm{PGL} / \mathrm{PHEO}$. The findings have significant implications for our understanding of CSS and CTr and for genetic counseling of both groups of patients.

\section{MATERIALS AND METHODS}

\section{Clinical studies}

A total of 63 unrelated patients were included in the study. Patients were seen mostly (but not exclusively) at the National Institutes of Health (NIH) Clinical Center from 1995 to the present time for evaluation and treatment of various tumors including PHEOS/PGLs. Diagnosis of all lesions was confirmed after surgical removal of the tumors and appropriate histology. The Institutional Review Boards of the participating institutions approved the studies: all patients provided written consents (and older children assented); those who were found to carry $S D H x$ defects were counseled accordingly. ${ }^{18}$

IHC was performed in fixed specimens obtained from surgically excised tumors of the studied patients, as published previously. ${ }^{8,13}$ The diagnoses of PGL, PHEO, GIST or CHO were made by established criteria using the appropriate antibodies, respectively. ${ }^{4,8,13}$

\section{Genetic studies}

DNA was obtained from peripheral blood lymphocytes (PBLs) or tumors, and extracted according to the manufacturer's protocol (Qiagen, Valencia, CA, USA). For germline DNA testing, deletion analysis and direct bidirectional sequencing were used to analyze all coding regions and the flanking exon/ intron junctions of the SDHA (OMIM: 600857, NM_004168.3), SDHB (OMIM: 185470, NM_003000.2), SDHC (OMIM: 602413, NM_003001.3), SDHD (OMIM: 602690, NM_003002.3) and SDHAF2 (OMIM: 613019, NM_017841.2) genes, by standard methods as described previously. ${ }^{8,13}$ All detected variations were submitted to ClinVar, a public database, and can be found in http://www.ncbi.nlm.nih.gov/clinvar/submitters/505422/ under the accession IDs SCV000222637-SCV000222642. The possible functional impact of an amino-acid change was predicted by two different in silico prediction tools: (a) the PolyPhen (Polymorphism Phenotyping) program (http://genetics. bwh.harvard.edu/pph2), which calculates the position-specific independent count score that represents the probability that a substitution is damaging; values nearer to 1 are more confidently predicted to be deleterious; (b) the Sorting Intolerant From Tolerant (SIFT) (http://sift.jcvi.org) calculates normalized probabilities for all possible substitutions from the alignment. Positions with normalized probabilities $<0.05$ are predicted to be deleterious; those $\geq 0.05$ are predicted to be tolerated. Prediction of splicing in silico was performed using the Berkeley Drosophila Genome Project (BDGP) (http:// www.fruitfly.org/segtools/splice.html) and the SplicePort analysis tool (http:// www.spliceport.org). Patients with known variations were excluded. All the variations detected were compared with the TCA Cycle Gene Mutation Database. $^{19}$

Tumor DNA studies were as published previously. ${ }^{5,13}$ In five patients with $\mathrm{CTr}$, we also obtained comparative genomic hybridization (CGH) of PBL DNA, with methods that we have recently published. ${ }^{20}$ There were no consistent or informative changes beyond what has already been published ${ }^{5}$ and these data are not shown.

Relatives of the patients were screened for these genes only in the presence of a sequence variant in the studied gene or if there was suggestive clinical history; patients with PHEO only were also screened for all known PHEO-causing genes (data not shown). All GISTs have also been sequenced at least for exons 9, 11, 13 and 19 of the KIT and exons 12 and 18 of the PDGFRA gene and found to be negative.

Statistical comparisons between the total cohort of patients with CTr and those with $S D H x$ variants were performed by $t$-test analysis for parametric data or $\chi^{2}$ analysis for non-parametric comparisons.

\section{RESULTS}

The patient age range at diagnosis was $8-50$ years (median, 23 years); 44 patients were 21 years old or younger. A total of $92 \%$ of the patients were female. GIST was located in the gastric area in all patients. Six patients $(9.5 \%)$ had germline variants in $S D H A, S D H B$ or $S D H C$ (Table 1). The median age in these six patients was 21 years, whereas three were females $(P<0.05$ compared with the total cohort).

Multiple synchronous GISTs were detected in $3 / 5$ patients. The median diameter of the dominant tumor was $3.5 \mathrm{~cm}(2-15 \mathrm{~cm})$. Histopathologic examination of GIST in all five patients showed reactivity for CKIT, wheres four had SDHB loss by IHC. Epithelioid histology was found in the GIST of three patients, whereas two had spindle histology in their GIST. The median mitotic rate per $50 \mathrm{hpf}$ was $7.5(5-14)$.

We were able to identify inheritance of PGL only in one family; there was no evidence of GIST (CTR-NIH593.01; Figure 1). The proband had incomplete CTr, with PGL and CHO (Figure 2). His mother and sister were carriers of the same SDHB defect, diagnosed with PGL/PHEO, and his nephew, also a carrier, was diagnosed with $\mathrm{NB}$ at the age of 3 years. A female patient with $\mathrm{CTr}$ and an SDHC mutation also had a ganglioneuroma, a tumor similar to NB.

Although PGLs or PHEOs were found in the six probands with CTr and $S D H x$ variants, none reported symptoms related with functional PGL/PHEO (palpitations, high blood pressure, excessive sweating). Measurement of plasma and 24-h urine catecholamines in five of the studied five patients revealed no abnormalities (in one patient this measurement was never done). Thus far, none of the carriers, relatives of the CTr patients, have developed any tumors other than PGL/PHEO.

Three of the sequence defects were missense substitutions that led to amino-acid changes, which are highly conserved across species. One splice site variant was identified in $S D H C$ at position +1 of intron 5 (c.405+1G > A). This appears to be a pathogenic mutation as has been reported previously in patients with PGL4 and in those with CSS, as well in those with sporadic SDH-deficient GIST; it is known to cause deletion of exon 5 and, therefore, results in a frameshift and premature termination of the protein. Two variants led to premature stop codons and are presumed pathogenic (Table 1). None of the variants was novel: all have been previously reported as affecting protein expression or function. None of the other $57 \mathrm{CTr}$ patients had mutations, deletions or other genomic defects affecting the $S D H x$ genes.

\section{DISCUSSION}

The present study of an expanded cohort of patients with CTr confirmed the data by Matyakhina et al: ${ }^{5}$ the vast majority of patients with $\mathrm{CTr}$ have no identifiable $S D H x$ coding sequence defects. Nevertheless, the study also showed that, rarely, SDHA, SDHB and SDHC defects can be found in selected patients with CTr. Were there any differences in these patients with CTr compared with the total NIH cohort, including the ones studied by Matyakhina et al? $?^{5}$ First, the 
similarities: they were equally young when they presented (at the age of 21 years versus 23 years for the whole cohort), their tumors were not more or less aggressive, and they are all alive at the time of this writing. However, unlike the rest of the CTr patients who are overwhelmingly female, those with $\mathrm{SDH} x$ defects were disproportionately male $(50 \%$ versus $8 \%, P<0.05)$ following the pattern of other diseases associated with $S D H x$ mutations that typically have no gender predilection..$^{9,18,21}$ The other interesting finding was the association with other neural crest tumors that have not previously been associated with CTr: ganglioneuroma in one patient with SDHC variation and $\mathrm{NB}$ in the nephew of another, in association with the same SDHB defect; in this family, no other patient had CTr: the obligate carriers had PGL or PHEO. In other words, in accordance with all known CTr patients in whom the disease appears sporadically, even in these patients where $S D H x$ mutations were present, CTr was never inherited in its full form.

A germline heterozygous missense $S D H B$ variant (SDHB, c.600G > T, p.(W200C)) in a patient with PHEO and a calcified lung nodule has been reported, but there was never biopsy of the lesion to confirm the diagnosis of $\mathrm{CHO}^{22}$ The combination of NB, PGL and renal cell carcinoma was first described by Fairchild et al. ${ }^{23}$ Most recently, the case of a patient who was found to have at the same time malignant NB, PHEO and renal cell carcinoma was described; sequence analysis revealed an $S D H B$ mutation. ${ }^{24}$ In another patient with NB and a PGL, a germline SDHB gene deletion was found. ${ }^{25}$ All in all, our two patients with $S D H x$ variants and family occurrence of $\mathrm{NB}$ or co-occurrence in the same patient of ganglioneuroma confirm the possibility of an association of SDH deficiency with these neural crest tumors. It is noteworthy that none of the other CTr patients (that had normal SDHx coding sequence) had a similar tumor, whereas $2 / 6$ (33.3\%) CTr patients with SDHx defects had such an association; one could speculate that this finding could reflect a higher predisposition of CTr patients with $S D H x$ germline variations to such neural crest lesions. Interestingly, too, only one of the $S D H x$ variants in the present study affected splicing of the corresponding gene; it is possible that this may represent a difference with CSS, in which a higher number of splicing $S D H x$ mutations has been identified.

What do the above tell us about the possible genetic defect(s) leading to CTr? CGH in tumors had previously shown copy number gains or losses in $1 \mathrm{q}, 1 \mathrm{p}$ and less frequently in the $14 \mathrm{q}$ and $22 \mathrm{q}$ chromosomal loci. ${ }^{5}$ Although the SDHB gene is located on chromosome 1p36.1-p35 and SDHC on 1q21-q23.3, deletions or coding sequence mutations had not been found. Now, the expanded CTr cohort confirms the involvement of the $S D H x$ genes at the germline level in a small number of CTr cases.

The first time that SDH deficiency was implicated in the pathogenesis of CTr was when we found lack of immunoreactivity for SDHB in CTr tumors that had normal SDHx sequence. ${ }^{8,13}$ The recent identification of SDHC-specific methylation ${ }^{14}$ established further the connection between SDH deficiency and CTr. However, one could only speculate on what may be the defect in the vast majority of CTr cases with normal SDHx sequence: it could be defects in a molecule involved in SDH function, from a noncoding RNA to a protein that has not been studied yet, or it could even be aberrant genomic architecture in the vicinity of the $S D H x$ genes that we have not detected using classic CGH or other whole-genome studies. It remains a mystery why the full spectrum of CTr is not inherited, even in the $S D H x$-mutated cases reported in the present study. This finding and the fact that the SDHx defects that we describe here have been reported previously in patients that did not have CTr suggest that perhaps multiple hits - beyond the known tissue-specific loss of the 

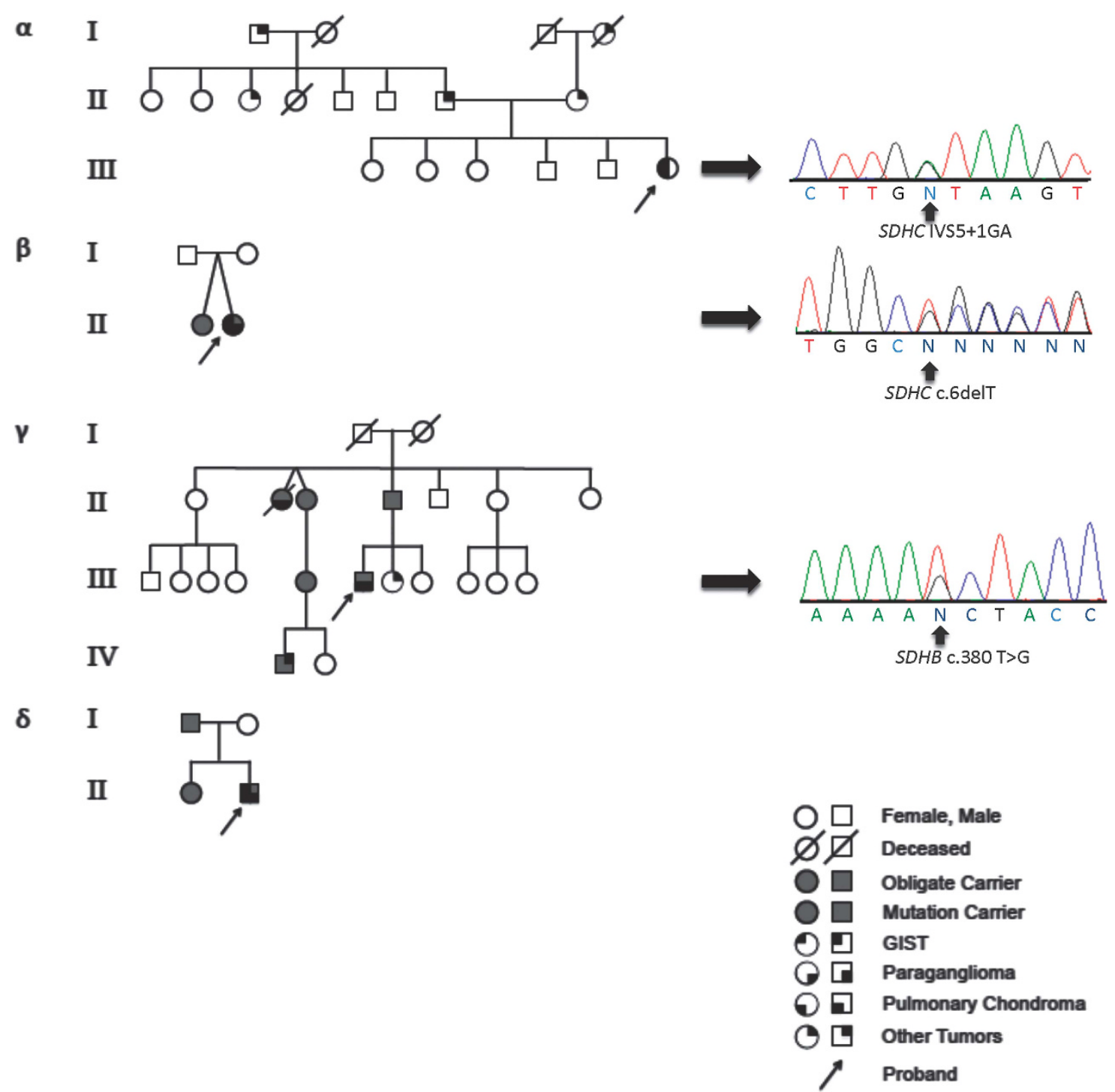

Figure 1 Families with $\mathrm{CTr}$ and SDH subunit gene mutations.

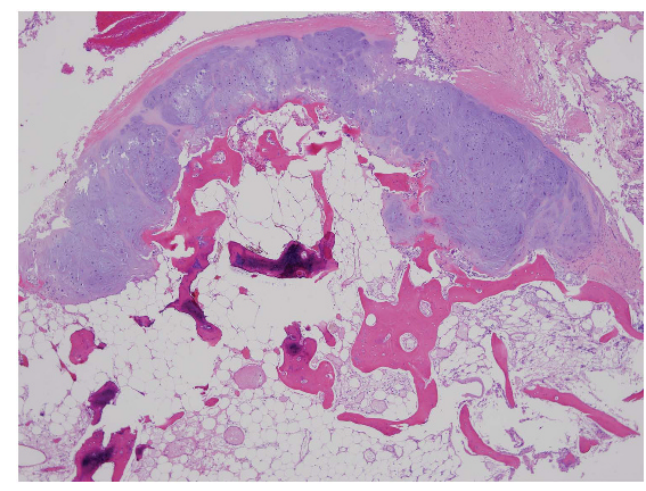

Figure 2 Pulmonary chondroma of the patient with $\mathrm{CTr}$ and a germline SDHB mutation. The lesion was composed of hypocellular cartilage undergoing central osseous and fatty metaplasia. It was surrounded by a thick fibrous capsule (hematoxylin and eosin staining, $\times 40$ ). normal SDHx allele - is needed to develop CTr; these additional hits may not be in the SDH enzyme but in other genes or genetic loci.

We conclude that CTr may, rarely, be associated with SDHx mutations that affect protein function. Although this finding does not change the fact that the vast majority of $\mathrm{CTr}$ patients get the disease from a yet elusive genetic defect, it does add significant information that needs to be considered in the genetic counseling of patients with $\mathrm{CTr}$ and their family members.

\section{CONFLICT OF INTEREST}

The authors declare no conflict of interest.

\section{ACKNOWLEDGEMENTS}

We thank the patients and their families for participating in our studies. This work was supported by the intramural research program of NICHD, NIH. 
1 Carney JA, Sheps SG, Go VL, Gordon H: The triad of gastric leiomyosarcoma, functioning extra-adrenal paraganglioma and pulmonary chondroma. $N$ Engl J Med 1977; 296: 1517-1518.

2 Boikos SA, Stratakis CA: The genetic landscape of gastrointestinal stromal tumor lacking KIT and PDGFRA mutations. Endocrine 2014; 47: 401-408.

3 Carney JA: Carney triad: a syndrome featuring paraganglionic, adrenocortical, and possibly other endocrine tumors. J Clin Endocrinol Metab 2009; 94: 3656-3662.

4 Carney JA, Stratakis CA, Young Jr WF: Adrenal cortical adenoma: the fourth component of the Carney triad and an association with subclinical Cushing syndrome. Am J Surg Pathol 2013; 37: 1140-1149.

5 Matyakhina L, Bei TA, McWhinney SR et al: Genetics of carney triad: recurrent losses at chromosome 1 but lack of germline mutations in genes associated with paragangliomas and gastrointestinal stromal tumors. J Clin Endocrinol Metab 2007; 92: 2938-2943.

6 Carney JA, Stratakis CA: Familial paraganglioma and gastric stromal sarcoma: a new syndrome distinct from the Carney triad. Am J Med Genet 2002; 108: 132-139.

7 McWhinney SR, Pasini B, Stratakis CAInternational Carney TCarney-Stratakis Syndrome C: Familial gastrointestinal stromal tumors and germ-line mutations. $N$ Engl J Med 2007; 357: 1054-1056

8 Janeway KA, Kim SY, Lodish M et al: Defects in succinate dehydrogenase in gastrointestinal stromal tumors lacking KIT and PDGFRA mutations. Proc Natl Acad Sci USA 2011; 108: 314-318.

9 Pasini B, Stratakis CA: SDH mutations in tumorigenesis and inherited endocrine tumours: lesson from the phaeochromocytoma-paraganglioma syndromes. J Intern Med 2009; 266: 19-42.

$10 \mathrm{Hao} \mathrm{HX}$, Khalimonchuk O, Schraders M et al: SDH5, a gene required for flavination of succinate dehydrogenase, is mutated in paraganglioma. Science 2009; 325: 1139-1142.

11 Pantaleo MA, Astolfi A, Indio V et al: SDHA loss-of-function mutations in KIT-PDGFRA wild-type gastrointestinal stromal tumors identified by massively parallel sequencing. $J$ Natl Cancer Inst 2011; 103: 983-987.

12 Stratakis CA, Carney JA: The triad of paragangliomas, gastric stromal tumours and pulmonary chondromas (Carney triad), and the dyad of paragangliomas and gastric stromal sarcomas (Carney-Stratakis syndrome): molecular genetics and clinical implications. J Intern Med 2009; 266: 43-52.
13 Gaal J, Stratakis CA, Carney JA et al: SDHB immunohistochemistry: a useful tool in the diagnosis of Carney-Stratakis and Carney triad gastrointestinal stromal tumors. Mod Pathol 2011; 24: 147-151.

14 Haller F, Moskalev EA, Faucz FR et al: Aberrant DNA hypermethylation of SDHC: a novel mechanism of tumor development in Carney triad. Endocr Relat Cancer 2014; 21: 567-577.

15 Killian JK, Miettinen M, Walker RL et al: Recurrent epimutation of SDHC in gastrointestinal stromal tumors. Sci Transl Med 2014; 6: 268ra177.

16 Letouze $\mathrm{E}$, Martinelli $\mathrm{C}$, Loriot $\mathrm{C}$ et al: SDH mutations establish a hypermethylator phenotype in paraganglioma. Cancer Cell 2013; 23: 739-752.

17 Killian JK, Kim SY, Miettinen M et al: Succinate dehydrogenase mutation underlies global epigenomic divergence in gastrointestinal stromal tumor. Cancer Discov 2013; 3: 648-657.

18 Raygada M, King KS, Adams KT, Stratakis CA, Pacak K: Counseling patients with succinate dehydrogenase subunit defects: genetics, preventive guidelines, and dealing with uncertainty. J Pediatr Endocrinol Metab 2014; 27: 837-844.

19 Bayley JP, Devilee P, Taschner PE: The SDH mutation database: an online resource for succinate dehydrogenase sequence variants involved in pheochromocytoma, paraganglioma and mitochondrial complex II deficiency. BMC Med Genet 2005; 6: 39.

20 Trivellin G, Daly AF, Faucz FR et al: Gigantism and acromegaly due to Xq26 microduplications and GPR101 mutation. N Engl J Med 2014; 371: 2363-2374.

21 Xekouki P, Szarek E, Bullova $\mathrm{P}$ et al: Pituitary adenoma with paraganglioma/ pheochromocytoma (3PAs) and succinate dehydrogenase defects in human and mice. J Clin Endocrinol Metab 2015; jc20144297.

22 Alrashdi I, Bano G, Maher ER, Hodgson SV: Carney triad versus Carney Stratakis syndrome: two cases which illustrate the difficulty in distinguishing between these conditions in individual patients. Fam Cancer 2010; 9: 443-447.

23 Fairchild RS, Kyner JL, Hermreck A, Schimke RN: Neuroblastoma, pheochromocytoma, and renal cell carcinoma. Occurrence in a single patient. JAMA 1979; 242 2210-2211.

24 Schimke RN, Collins DL, Stolle CA: Paraganglioma, neuroblastoma, and a SDHB mutation: resolution of a 30-year-old mystery. Am J Med Genet Part A 2010; 152A 1531-1535.

25 Armstrong R, Greenhalgh KL, Rattenberry E et al: Succinate dehydrogenase subunit B (SDHB) gene deletion associated with a composite paraganglioma/neuroblastoma. J Med Genet 2009; 46: 215-216. 